Meta

Journal des traducteurs

Translators' Journal

\title{
Saint-John Perse : le paradoxe de l'hermétisme et de la traductibilité
}

\section{Nebil Radhouane}

Volume 45, numéro 3, septembre 2000

La traduction dans le monde arabe

URI : https://id.erudit.org/iderudit/003903ar

DOI : https://doi.org/10.7202/003903ar

Aller au sommaire du numéro

Éditeur(s)

Les Presses de l'Université de Montréal

ISSN

0026-0452 (imprimé)

1492-1421 (numérique)

Découvrir la revue

Citer cet article

Radhouane, N. (2000). Saint-John Perse : le paradoxe de l'hermétisme et de la traductibilité. Meta, 45(3), 445-449. https://doi.org/10.7202/003903ar

\section{Résumé de l'article}

Prenant comme point de départ une poésie réputée hermétique comme celle de Saint-John Perse et confrontant plusieurs traductions en arabe, l'auteur montre comment une lecture de cette poésie dans "sa modulation globale", c'est-à-dire "dans ses notes, non dans ses mots", arrive à une traduction qui préserve cette "universalité programmée par Perse dans la syntaxe et non dans le lexique". 


\title{
Saint-John Perse: le paradoxe de l'hermétisme et de la traductibilité
}

\author{
NEBIL RADHOUANE \\ Université de Tunis I, Tunis, Tunisie
}

\begin{abstract}
RÉSUMÉ
Prenant comme point de départ une poésie réputée hermétique comme celle de SaintJohn Perse et confrontant plusieurs traductions en arabe, l'auteur montre comment une lecture de cette poésie dans «sa modulation globale», c'est-à-dire «dans ses notes, non dans ses mots», arrive à une traduction qui préserve cette «universalité programmée par Perse dans la syntaxe et non dans le lexique».
\end{abstract}

\begin{abstract}
Based on a poetry known for its hermetic quality such as that of Saint-John Perse, and comparing several Arabic translations, the author shows how the reading of this poetry in its "global modulation," that-is-to say "in its consonance, and not its words," can result in a translation that preserves this "universality introduced by Perse in the syntax rather than the lexis."
\end{abstract}

\section{MOTS-CLÉS/KEYWORDS}

Saint-John Perse, poésie, traductibilité, hermétisme, arabe.

Le poète Saint-John Perse n'aimait pas qu'Alain Bosquet imputât son audience à «traductibilité». Il contestait même jusqu'à la légitimité de cette forgerie:

Oui, l'étude de Bosquet est intelligente et méritait une publication autre que celle d'une collection de vulgarisation [...]. Je pense comme vous que sa théorie de l'image [...] ne répond pas à ce qui me concerne; [...] Son développement sur la traductibilité (le mot est-il français?) de mon œuvre m'a paru aussi mal justifié, car on ne peut être plus traduisible qu'en donnant - et en français! - toute l'importance que je donne à la langue, à sa métrique interne, à son essence, à la substance même du mot ou à sa dérivation. Si j'ai pu tenter quelques traducteurs (et je n'ai jamais compris pourquoi), c'est certainement en dépit de la difficulté de traduction ${ }^{1}$.

C'est pourtant cette «traductibilité» de la poésie persienne — que le poète «ne comprenait pas» — qui fut l'une de ses voies royales à l'universalité. Qu'il s'agisse d'une traductibilité infuse dans l'œuvre, et dont même Saint-John Perse ignorait le secret, ou d'une technique d'écriture sciemment mobilisée comme autre argument de la stratégie et de la rhétorique persiennes, la postérité aura démenti les présomptions du poète prix Nobel quant à l'essence «on ne peut plus intraduisible» de son œuvre. «En dépit» du lexique, que d'aucuns tiendraient pour l'un des plus obscurs et des plus spécialisés de la littérature française, la poésie persienne a passé les frontières linguistiques avec une rapidité et une aisance si étonnante pour une œuvre réputée hermétique.

Du reste, «la difficulté de traduction» invoquée par Saint-John Perse est en vérité un problème générique et non particulier à sa poésie. Ses réticences sont celles-là 
même qu'aurait exprimées tout poète jaloux et de son œuvre et de sa langue natale. Qui des poètes ne l'est pas? Les écrivains les mieux traduits sont, dans le secret du cœur, fondamentalement convaincus de cette sorte d'expropriation artistique sous la bannière de la traduction. Quelle que soit la gloire dont ils bénéficient par la grâce de cette migration linguistique heureuse et gratifiante, ils ne pourront jamais y souscrire sans réprimer un sentiment de gêne, voire d'envie, envers ce créateur de seconde main qu'est le traducteur. C'est donc cet ascendant de l'écrivain, indûment revendiqué par rapport à son traducteur, qui est aussi connoté par la célèbre formule de Dominique Aury (Mounin 1963: VII) :

Dans l'armée des écrivains, nous autres traducteurs nous sommes la piétaille.

À l'autre bout, le traducteur doit alors éprouver la même gêne et la même envie, lui qui s'adosse à la création d'autrui et ne peut que la répéter - au prix de quelles trahisons (heureusement!) car c'est la seule marge de création et d'empreinte personnelle où il lui soit possible de se mouvoir librement.

On ne doit donc pas infirmer la thèse de la traductibilité - si justement pressentie par Bosquet - sur la seule foi du poète, dont les arguments sont au surplus génériques et donneraient pour ainsi dire raison à tous les poètes adoptant cette attitude. Quand Saint-John Perse parle de «substance même du mot» et de «sa dérivation», parle-t-il d'autre chose que de ce qui devrait être «trahi» dans toute traduction digne de ce nom? Une traduction poétique pourrait-elle même se réaliser sans la trahison du signifiant de départ? Admettons avec Olivier Reboul (1984:37) que «la première caractéristique des figures de mots est qu'elles sont intraduisibles, à moins qu'on ne trouve par hasard leur équivalent dans une autre langue».

Or, contre quoi Saint-John Perse met-il en garde ses traducteurs sinon contre la trahison - du reste incontournable en toute traduction — de la face palpable du poème? En déplorant cette sorte d'expatriation forcée de la «substance même du mot» et de «sa dérivation», qu'est-ce qu'il regrette sinon ces «figures de mots» intransposables d'une langue à l'autre et d'ailleurs «non paraphrasables» même à l'intérieur de la langue d'origine?

\section{Des traductions arabes de l'œuvre persienne}

Le choix de l'arabe semblerait réducteur, mais la langue de Jahiz pourrait justement, à titre échantillonnaire, constituer une illustration extrême de la «traductibilité» persienne. Langue réputée difficile, elle serait par conséquent assez représentative de cette capacité de l'œuvre persienne à migrer aisément dans toutes les langues et à franchir les barrières linguistiques quelles qu'elles soient. Par «difficile», il ne faudrait entendre aucune échelle de valeur établie entre les langues, mais une « expatriation» et un "dépaysement» autrement plus perceptibles. Lisons à ce sujet telle précision du poète syro-libanais Adonis (1994: 8):

J'ajouterai que la transposition d'une langue à une autre, radicalement différente, comme celle du français à l'arabe ou de l'arabe au français, est un véritable déracinement. Ce n'est pas une simple visite familiale, comme c'est le cas pour les langues unies par des racines communes qui font qu'elles ne constituent pratiquement qu'une seule langue. 
Dans le numéro 3 de la revue Souffle de Perse, paru en janvier 1993, nous avions déjà déploré qu'en France on n'eût aucune connaissance de la traduction du Tunisien Ali Louati, qui non seulement réalisa l'une des plus honnêtes transpositions arabes d'Anabase, d'Exil et du Discours de Stockholm, mais eut le mérite de relever plusieurs erreurs inadmissibles dans la traduction d'Adonis qui, réelle méprise ou ménagement dû à la célébrité, demeure le seul traducteur persien reconnu en France. Certes, il existe une troisième traduction, aussi solide que celle de Louati, mais pour d'aucuns plus attrayante (parce qu'elle se conforme aussi bien à toutes les fidélités souhaitées qu'à toutes les trahisons permises!): celle du Marocain Mustapha El Kasri. Cette adaptation d'inspiration coranique eut certainement les vents plus favorables que celle de Louati, mais elle demeure toujours moins connue en France que celle d'Adonis (El Kasri 1972; voir aussi Hogga 1984: 14-29, avec une note du traducteur, p. 11-12).

$\mathrm{Ne}$ citant que les traductions d'Adonis, la prestigieuse édition La Pléiade va même jusqu'à confondre ce dernier avec Yussuf Al Khal. On sait que le vrai nom d'Adonis est Ali Ahmed Saïd; nous lisons à la page 1365 des Euvres complètes, à propos de la traduction arabe d'Étroits sont les vaisseaux:

Traduction de Yussuf Al Khal (Adonis) [ sic] dans la revue Shi'r, Beyrouth, vol. III, $\mathrm{n}^{\circ}$ 4, automne 1957.

Nous avions dénoncé cette confusion dans Souffle 3 en nous demandant si c'est Adonis qui a été pris pour Yussuf $\mathrm{Al}$ Khal ou Yussuf $\mathrm{Al}$ Khal pour Adonis, question à laquelle nous aurons trouvé réponse dans le numéro 4 de Souffle grâce au Marocain Azeddine Khaloufi. En s'appuyant sur une lettre datée du 29 juin 1960, où Yussuf Al Khal, qui était alors le fondateur de la revue Shi'r, demandait la permission à SaintJohn Perse de traduire Amers en arabe, Khaloufi affirme que le projet était celui d'Adonis, qui co-dirigeait la revue libanaise. La confusion venait donc de ce que Yussuf Al Khal, en demandant cette autorisation, parla à la première personne du pluriel et, aux dires de Azeddine Khaloufi, qui aurait consulté la lettre originale à la Fondation Saint-John Perse d'Aix-en-Provence ${ }^{2}$, écrivit «dans un anglais assez médiocre» :

We are planning now, after your permission of course, to translate all of Amers into Arabic, to be published in book from. (Khaloufi 1994: 15)

Quoique partiellement résolu, ce problème mérite le détour parce qu’il lève le voile sur un état arbitraire de la consécration officielle. Que les erreurs de traduction commises par Adonis trouvent des justifications théoriques et poétiques, on l'admet volontiers, mais que l'on aille jusqu'à se tromper de personnes explique que l'on se soit si souvent trompé de mérite et de valeur. Dès lors, il n'est plus du tout surprenant que le lecteur français, sous le prétexte d'ignorer la langue arabe, invoque la seule notoriété du nom d'Adonis comme argument d'autorité, sans jamais se soucier de contrôler la valeur du traducteur ni de sa traduction.

\section{La traductibilité de Perse: l'exemple d'Adonis}

Nous ne reviendrons pas sur les erreurs d'Adonis, lesquelles ont été énumérées par Louati dans un article intitulé "La mise à mort d'un discours poétique, Adonis bourreau de Saint-John Perse ${ }^{3} »$. Mais nous montrerons cette fois comment, sans faire 
exprès, le poète syro-libanais aura souvent l'intuition, poétiquement fidèle, de traduire le mouvement sans se soucier du lexique. Il lui arrivera même de faire mieux que Louati, auquel Shlomo Elbaz eût fait le même reproche qu'à Eliot comparé à Little donnant du verset persien suivant:

Les armes au matin sont belles et la mer. (Perse 1960: 93; c'est nous qui soulignons)

les transpositions anglaises respectives:

Beautiful are bright weapons in the morning and behind us the sea is fair Weapons by morning are lovely and the sea

Comme le souligne Elbaz (1977), la première traduction, celle de T. S. Eliot, est lexicalement saturée, ce qui dessert l'élan elliptique de l'original : fair répète beautiful, bright est superfétatoire, et le locatif behind us réduit le champ sémantique autrement plus ouvert, plus suspendu, dans le mystère de l'ellipse.

Celle de Little tiendra compte, quant à elle, du raccourci verbal et de l'extrême compression syntaxique inversement proportionnelle à l'expansion sémantique de la séquence et la mer.

Curieusement, dans les transpositions arabes Louati fera comme Eliot et Adonis comme Little. En voici les traductions respectives:

[?al?aslihatu zami:latun fis șaba:hi wazami:lun huwalbahru] (Louati $1985: 103$ )

[?alPaslihatu walbahru zami:latun fisssaba:h] (Adonis 1978)

Certes Louati s'en sort syntaxiquement mieux qu'Eliot, dans la mesure où il répète tel quel l'adjectif et récupère ainsi une réduplication toute persienne, disposée de surcroît en chiasme, cependant que le poète anglais a répété l'adjectif par un synonyme approchant. Toutefois, dans les deux cas, l'ellipse est sacrifiée.

On pourrait toujours reprocher à Adonis ses traductions lexicales fautives et qui frisent quelquefois le contresens, comme en témoigne dans la traduction de l'incipit d'Étroits sont les vaisseaux, la transposition du mot amants par [üahibbaü]. Le poètetraducteur n'a cependant pas oublié de respecter le parallélisme anaphorique au mépris de la cécité lexicale:

[?ajjuha :1Rahibba? ... Rajjuha :lRahibbaü] (Adonis 1976: 93)

Beaucoup d'autres exemples ${ }^{4}$ dans les traductions adonisiennes confirment ainsi cette traductibilité que contestait le poète français et que le poète syro-libanais illustrait tant et si bien malgré sa connaissance approximative de la langue de Molière.

Une fois prouvée, cette traductibilité de Perse constitue par elle-même un paradoxe: comment une ouvre au lexique si peu courant en français a-t-elle pu franchir les barrières inter-linguistiques alors qu'il n'était pas toujours évident qu'elle franchît tous les obstacles intra-linguistiques? L'énigme s'élucide au travers d'une stratégie de l'universalité programmée par Perse dans la syntaxe et non dans le lexique.

La confrontation des deux traductions, celle de Louati et celle d'Adonis, a permis de prouver que plus on est proche de la langue natale du poète, moins on sent l'universalité de sa syntaxe. À son insu, Adonis fut ce lecteur modèle qui dut percevoir cet aspect universel - figural plus que grammatical - de la syntaxe persienne. En d'autres termes, c'est dans sa modulation globale qu'il a lu cette poésie, et non dans sa langue, dans ses notes ${ }^{5}$, non dans ses mots. 
Inversement, comme un lecteur natif, Louati n'a pu percevoir le texte persien dans cette «exemption de sens». Les mots l'ont d'abord intéressé et sa traduction, pour avoir été fidèle au sens littéral — et lexicalement beaucoup trop lucide — s'est engluée dans la langue et la grammaire.

Il ne s'agit nullement de préférer la traduction d'Adonis à celle de Louati. Ce dernier avait à juste raison dénoncé plusieurs fautes graves chez le premier et, en divers endroits, sa traduction demeure bien meilleure. Toutefois, très fidèle au sens littéral — ce qui est du reste souhaitable mais non primordial — et résolument plus grammairien dans ses traductions que poète, Louati aurait pu ne pas s'écarter de la syntaxe de départ. Celle-ci n'avait qu'à être transposée telle quelle et autorisait même qu'on la confortât dans sa rythmique originale.

\section{NOTES}

1. Lettre à Roger Caillois, du 25 juillet 1953, La Pléiade, p. 967-968. C’est nous qui soulignons. SaintJohn Perse y confie ses impressions sur le livre que lui consacra Bosquet (1977).

2. Khaloufi 1994: 14-15. Khaloufi écrit (p. 14): «Nous avons consulté la lettre originale à la Fondation Saint-John Perse à Aix-en-Provence.»

3. C'est nous qui traduisons. Le procès de Louati paraît d'abord dans Horizons arabes, 2 (oct. 1980) puis dans Anabase, Exil et autres poèmes (1985: 135-157).

4. Ces exemples ont été relevés dans le cadre de notre thèse d'État La syntaxe dans l'œuvre poétique de Saint-John Perse, soutenue le 11 juin 1998 à l'Université de Tunis I.

5. Au sens musical s'entend.

\section{REFERENCES}

Bosquet, A. (1977): Saint-John Perse, Paris, Seghers, coll. «Poètes d'aujourd'hui ».

El Kasri, M. (1972): Étroits sont les vaisseaux, préf. Mohamed Mzali, Tunis, Maison tunisienne d'edition.

Hogga, M. (1984): «Une traduction d'Amers en arabe», extraits choisis du chapitre IX, Cahiers Saint-John Perse, 7.

Elbaz, S. (1977): Lectures d'Anabase de Saint-John Perse, le désert, le désir, L'Âge d'Homme.

Khaloufi, A. (1994) : "Adonis traducteur arabe de Saint-John Perse», Souffle de Perse, 4, Revue de l'Association des Amis de la Fondation Saint-John Perse.

Louati, A. (1985): Anabase, Exil et autres poèmes, Maison arabe du livre.

Mounin, G. (1963): Les problèmes théoriques de la traduction, préf. Dominique Aury, Paris, Gallimard.

Reboul, O. (1984): La rhétorique, Paris, Presses universitaires de France, coll. "Que sais-je?», $\mathrm{n}^{\circ} 2133$.

SAÏD, Ali A. dit Adonis (1976): Amers, Manarat, Damas, Éditions du Ministère de la Culture.

— (1978): Euvres complètes, Exil et autres poèmes, Damas, Éditions du Ministère de la Culture.

— (1994): «Conférence d’Adonis donnée à la Fondation Saint-John Perse le 9 octobre 1993, traduite de l'arabe par Anne Wade Minkowski en collaboration avec l'auteur», Souffle de Perse, 4, Revue de l'Association des Amis de la Fondation Saint-John Perse.

Saint-John Perse (1960): Euvres complètes, Paris, Gallimard, coll. «La Pléiade» (1960 pour l'édition revue et corrigée; 1972 pour la biographie, les notices, les notes, les textes inédits et la bibliographie; 1982 pour les derniers poèmes Nocturne et Sécheresse). 